\title{
An Inventory Model for Solving Two Stage Supply Chain using Fuzzy Costs with Shortage
}

\author{
P. Parvathi \\ Head \& Associate Professor, \\ Department of Mathematics, \\ Quaid- E -Millath Government College for Women \\ (Autonomous), \\ Chennai-600002
}

\author{
S. Gajalakshmi \\ Research Scholar, \\ Department of Mathematics, \\ Quaid- E -Millath Government College for Women \\ (Autonomous), \\ Chennai-600002
}

\begin{abstract}
A fuzzy inventory model is proposed to maximize the profit in a two stage supply chain model. In this paper joint total profit of both buyer and vendor are calculated. Shortage for the buyer is allowed and it is completely backlogged. Number of shipments, selling price and order quantity are taken as decision variables. Graded mean integration representation method is applied for defuzzification. Sensitivity analysis is carried out using the numerical example.
\end{abstract}

Keywords: Linear demand, vendor - buyer coordination, fuzzy numbers and fuzzy concepts

\section{INTRODUCTION}

In last few years, companies have realized that efficient management of inventories across the different facilities in a supply chain is critical to increase the profits. This efficient management is achieved through better co-ordination and more co-operations between the vendor and the buyer. The supply chain management has enabled numerous firms to enjoy great advantages by integrating all activities associated with the flow of material, information, and capital between suppliers of raw materials and the ultimate customers. The benefits of a properly managed supply include reduced cost, faster product delivery, greater efficiency and lower cost for both the business and its customers.

In traditional inventory Models, the inventory and shipment polices for the vendor and buyer in a two-echelon supply chain are taken independently. The optimal lot size for the buyer may not result in an optimal policy for the vendor and vice versa. To overcome this situations the integrated vendor - buyer model has been developed using fuzzy concepts. Determining the ordering and shipment polices with shortages allowed for buyer results in a reduction of the total inventory cost of the system if the determination is based on the integrated total cost function rather than the buyer's or vendor's individual cost function.

Goyal (3) early developed the idea of a joint total cost for a single vendor and single buyer scenario assuming an infinite production rate for the vendor and lot-for-lot policy for the shipments from the vendor to the buyer. Goyal (4) introduced a model where the shipment size increases by a factor equal to the ratio of the production rate to the demand rate. $\mathrm{He}$ formulated the problem and developed an optimal expression for the first shipment size as a function of number of shipments.

Lau and Lau [5] framed a joint-pricing inventory model with out setup cost. Ray, et al., [6] introduced a integrated marketing inventory model for two pricing policies, price as a decision variable and mark-up pricing. R. Akbari jokar [7] developed joint model to determine the profit function of buyer and vendor.

A. Nagoor Gani and G. Sabarinathan [8] developed fuzzy integrated inventory model to determine the relevant profit maximizing decision variable values. They did not allow shortages which is unrealistic. To suit the real life situation we allow shortages for both vendor and buyer. The final demand for the product is assumed to be deterministic but price sensitive. Production rate, ordering quantity, setup cost, shortage cost and holding cost of the buyer and vendor are taken as fuzzy numbers. The lot delivered from the vendor to the buyer is equal - sized batches. As soon as the on-hand inventory at the buyer drops to reorder point, an order of size $\tilde{Q}$ is released by the buyer. The vendor manufactures the product at the production rate $\tilde{P}$ and in lot sizes which are a multiple of $\tilde{Q}$. The objective is to determine the number of shipments, the selling price $\tilde{\delta}$ as well as order size by allowing shortages for buyer, so that the total profit of the vendor - buyer are maximized.

\section{METHODOLOGY}

\subsection{Fuzzy Numbers}

Any fuzzy subset of the real line $\mathrm{R}$, whose membership function $\mu_{\mathrm{A}}$ satisfied the following conditions, is a

generalized fuzzy number $A$

(i) $\mu_{\mathrm{A}}$ is a continuous mapping from $\mathrm{R}$ to the closed interval $[0,1]$,

(ii) $\mu_{\mathrm{A}}=0,-\infty<x \leq a$

(iii) $\mu_{\mathrm{A}}=\mathrm{L}(\mathrm{x})$ is strictly increasing on $\left[\mathrm{a}_{1}, \mathrm{a}_{2}\right]$

(iv) $\mu_{\mathrm{A}}=\mathrm{w}_{\mathrm{A}}, a_{2} \leq x \leq a_{3}$

(v) $\mu_{\mathrm{A}}=\mathrm{R}(\mathrm{x})$ is strictly decreasing on $\left[\mathrm{a}_{3}, \mathrm{a}_{4}\right]$

(vi) $\mu_{\mathrm{A}}=0, a_{4} \leq x \leq \infty$

Where $0<\mathrm{w}_{\mathrm{A}} \leq 1$ and $\mathrm{a}_{1}, \mathrm{a}_{2}, \mathrm{a}_{3}$ and $\mathrm{a}_{4}$ are real numbers. Also this type of generalized fuzzy number be denoted as $\widetilde{A}=\left(a_{1}, a_{2}, a_{3}, a_{4} ; w_{A}\right)_{L R} ;$ when $\mathrm{w}_{\mathrm{A}}=1$, it can be simplified as $\tilde{A}=\left(a_{1}, a_{2}, a_{3}, a_{4} ; w_{A}\right)_{L R}$.

\subsection{Triangular fuzzy number}

The fuzzy set $\tilde{A}=\left(a_{1}, a_{2}, a_{3}\right)$ where $\mathrm{a}_{1}<\mathrm{a}_{2}<\mathrm{a}_{3}$ and defined 
$\mathrm{R}$, is called the triangular fuzzy number, if the membership function of $\tilde{A}$ is given by

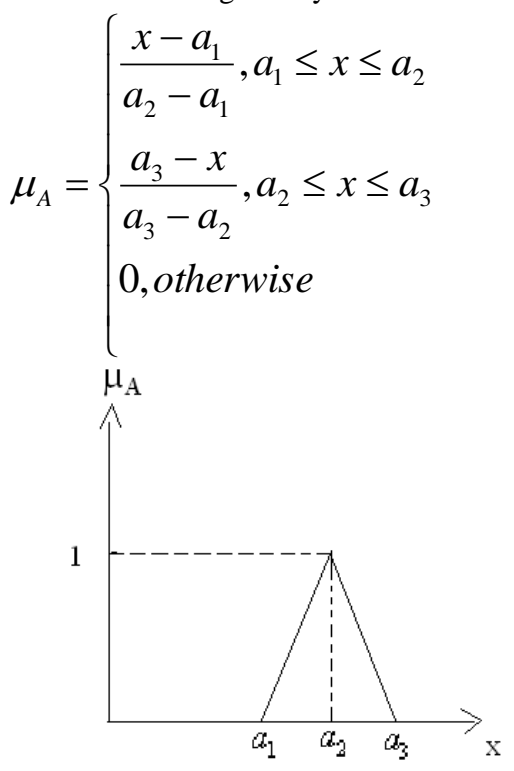

\subsection{The Function Principle}

The function principle was introduced by Chen [6] to treat fuzzy arithmetical operations. This principle is used for the operation for addition, subtraction, multiplication and division of fuzzy numbers.

$$
\text { Suppose } \quad \tilde{A}=\left(a_{1}, a_{2}, a_{3}\right) \quad \text { and }
$$

$\widetilde{B}=\left(b_{1}, b_{2}, b_{3}\right)$ are two triangular fuzzy numbers. Then

(i) The addition of $\tilde{A}$ and $\tilde{B}$ is

$$
\widetilde{A}+\widetilde{B}=\left(a_{1}+b_{1}, a_{2}+b_{2}, a_{3}+b_{3}\right) \quad \text { where }
$$

$a_{1}, a_{2}, a_{3}, b_{1}, b_{2}, b_{3}$ are any real numbers.

(ii) The multiplication of $\tilde{A}$ and $\tilde{B}$ is $\widetilde{A} \times \widetilde{B}=\left(c_{1}, c_{2}, c_{3}\right)$

Where

$T=\left(a_{1} b_{1}, a_{1} b_{3}, a_{3} b_{1}, a_{3} b_{3}\right), c_{1}=\min T, c_{2}=a_{2} b_{2}, c_{3}=\max$ if $a_{1}, a_{2}, a_{3}, b_{1}, b_{2}, b_{3}$ are all non zero positive real numbers, then $\widetilde{A} \times \widetilde{B}=\left(a_{1} b_{1}, a_{2} b_{2}, a_{3} b_{3}\right)$.

(iii) $-\widetilde{B}=\left(-b_{3},-b_{2},-b_{1}\right)$ then the subtraction of $\tilde{B}$ from $\tilde{A}$ is $\tilde{A}-\tilde{B}=\left(a_{1}-b_{3}, a_{2}-b_{2}, a_{3}-b_{1}\right)$ where $\mathrm{a}_{1}, \mathrm{a}_{2}, \mathrm{a}_{3}, \mathrm{~b}_{1}, \mathrm{~b}_{2}, \mathrm{~b}_{3}$ are any real numbers.

(iv) $\frac{1}{\widetilde{B}}=\widetilde{B}^{-1}=\left(\frac{1}{b_{3}}, \frac{1}{b_{2}}, \frac{1}{b_{1}}\right)$ where $\mathrm{b}_{1}, \mathrm{~b}_{2}, \mathrm{~b}_{3}$ are all non zero positive real numbers, then the division of $\tilde{A}$ and $\widetilde{B}$ is $\frac{\tilde{A}}{\widetilde{B}}=\left(\frac{a_{1}}{b_{3}}, \frac{a_{2}}{b_{2}}, \frac{a_{3}}{b_{1}}\right)$

$$
\begin{aligned}
& \text { (v) } \begin{array}{c}
\text { For } \\
K \tilde{A}
\end{array}=\left(K a_{1}, K a_{2}, K a_{3}\right) i f K>0 \\
& K \tilde{A}=\left(K a_{3}, K a_{2}, K a_{1}\right) i f K<0
\end{aligned}
$$

number

$\mathrm{K}$,

\subsection{Signed Distance Method}

Defuzzification of $\tilde{A}$ can be found by signed distance method. If $A$ is a triangular fuzzy number and is fully determined by $\left(a_{1}, a_{2}, a_{3}\right)$, the signed distance from

$A$ to 0 is defined as

$$
d(\tilde{A}, \tilde{0})=\int_{0}^{1} d\left(\left[A_{L}(\alpha), A_{R}(\alpha)\right], \tilde{0}\right) d \alpha=\frac{\left(a_{1}+4 a_{2}+a_{3}\right)}{4}
$$

\subsection{Notations}

the vendor

Q

buyer

$\begin{array}{ll} & \mathrm{A}_{\mathrm{v}} \\ \text { vendor } & \mathrm{A}_{\mathrm{b}} \\ & \mathrm{c}\end{array}$

purchasing price

the buyer

$$
\delta
$$

function of unit selling price

$$
\mathrm{h}_{\mathrm{v}}
$$

cost for the vendor per year

$$
\mathrm{h}_{\mathrm{b}}
$$

cost for the buyer per year$$
\mathrm{n}
$$$$
\mathrm{S}_{\mathrm{b}}
$$

buyer

$S$

level for the buyer

$\vec{P}=\left(P_{1}, P_{2}, P_{3}\right)$

of the vendor

$\tilde{Q}=\left(Q_{1}, Q_{2}, Q_{3}\right)$

Production rate of

Order quantity of the Setup cost of the Ordering cost of the the buyer's unit Unit selling price of Demand rate as a inventory holding inventory holding Number of shipments Shortage cost for the maximum inventory fuzzy production rate fuzzy order quantity of the buyer $\tilde{A}_{v}=\left(A_{v_{1}}, A_{v 2}, A_{v_{3}}\right)$ fuzzy setup cost of the vendor

$$
\widetilde{A}_{b}=\left(A_{b_{1}}, A_{b 2}, A_{b_{3}}\right)
$$

Fuzzy ordering cost of the buyer $\tilde{\delta}=\left(\delta_{1}, \delta_{2}, \delta_{3}\right)$ fuzzy unit selling price of the buyer $\tilde{D}=\left(D_{1}, D_{2}, D_{3}\right)$ Fuzzy demand rate as a function of unit selling price $\tilde{h}_{v}=\left(h_{v_{1}}, h_{v 2}, h_{v_{3}}\right) \quad$ - $\quad$ Fuzzy inventory holding cost for the vendor per year 
$\tilde{h}_{b}=\left(h_{b_{1}}, h_{b 2}, h_{b_{3}}\right) \quad-\quad$ Fuzzy inventory holding cost for the buyer per year $\tilde{n}=\left(n_{1}, n_{2}, n_{3}\right) \quad$ - number of shipments $\tilde{S}_{b}=\left(S_{b_{1}}, S_{b 2}, S_{b_{3}}\right) \quad$ - $\quad$ Fuzzy inventory shortage cost for the buyer per year $T \tilde{P}_{v}$ Annual profit function for the vendor $T \tilde{P}_{B}$ Annual profit function for the buyer

\subsection{Assumptions}

(i) The model deals with a single vendor and a single buyer for a single product.

(ii) The buyer faces a linear Demand $\widetilde{D}(\tilde{\delta})$ as a function of selling price $\tilde{\delta}$.

(iii) A finite production rate for the vendor is considered which is greater than the demand rate.

(iv) The inventory is continuously reviewed. The buyer orders a lot of size $\widetilde{Q}$ when the on-hand inventory reaches the reorder point.

(v) The vendor manufactures a production batch $n \tilde{Q}$ at one setup. However, the size of shipment delivered to the buyer is $\tilde{Q}$.

(vi) The inventory holding cost at the buyer is higher than that at the vendor. i.e., $\tilde{h}_{b}>\tilde{h}_{v}$

(vii) Shortage is allowed for buyer.

(viii) The time horizon is infinite.

\section{Fuzzy Mathematical Model}

The optimal policy of the integrated system is derived. However, for comparative purposes, we first obtain the buyer and the vendor policies, if each party optimizes its profit independently. The policies and profits are then compared to the case of integrated system when they cooperate, particularly in information sharing.

We assume that the buyer faces a linear demand $\tilde{D}(\tilde{\delta})=a-b \tilde{\delta}(\mathrm{a}>\mathrm{b}>0)$ as a function of its unit selling price. As $\tilde{D}(\tilde{\delta})>0$, the maximum selling price is a/b, i.e., $\tilde{\delta}<\frac{a}{b}$. The buyer's yearly profit is equal to the gross revenue minus the sum of purchasing, order processing, and inventory holding costs. The buyer wishes to maximize its yearly profit function, $T \widetilde{P}_{B}$ through the optimal choice of selling price and order quantity, i.e.,

$$
\begin{aligned}
& \tilde{T} P_{B}(\tilde{\delta}, \tilde{Q})=(a-b \tilde{\delta})(\tilde{\delta}-c) \\
& -\frac{\tilde{h}_{b} \tilde{S}^{2}}{2 \tilde{Q}}-\frac{\tilde{S}_{b}(\tilde{Q}-\tilde{S})^{2}}{2 \tilde{Q}}-\frac{\tilde{A}_{b}(a-b \tilde{\delta})}{\tilde{Q}}
\end{aligned}
$$

where

$$
\begin{aligned}
& \tilde{Q}=\sqrt{2 \tilde{A}_{b}(a-b \tilde{\delta}) \frac{\tilde{h}_{b}+\tilde{S}_{b}}{\tilde{h}_{b} \tilde{S}_{b}}} \\
& \tilde{S}=\frac{\tilde{Q} \tilde{S}_{b}}{\tilde{h}_{b}+\tilde{S}_{b}}
\end{aligned}
$$

Substituting equation (3) in equation (1), we get,

$$
\begin{aligned}
& \tilde{T} P_{B}(\tilde{\delta}, \tilde{Q})=(a-b \tilde{\delta})(\tilde{\delta}-c)-\frac{\tilde{h}_{b} \tilde{S}_{b}^{2} \tilde{Q}}{2\left(\tilde{h}_{b}+\tilde{S}_{b}\right)^{2}} \\
& -\frac{\tilde{S}_{b} \tilde{Q}\left(1-\frac{\tilde{S}_{b}}{\tilde{h}_{b}+\tilde{S}_{b}}\right)^{2}}{2}-\frac{\tilde{A}_{b}(a-b \tilde{\delta})}{\tilde{Q}}
\end{aligned}
$$

Substituting equation (2) in (1) we get,

$$
\begin{aligned}
& T \tilde{P}_{B}(\tilde{\delta})=\left(a \tilde{\delta}-b \tilde{\delta}^{2}-a c+b c \tilde{\delta}\right)- \\
& \sqrt{(a-b \tilde{\delta})}\left\{\begin{array}{l}
\frac{\tilde{h}_{b} \tilde{S}_{b}^{2} \sqrt{2 \tilde{A}_{b}} \sqrt{\frac{\tilde{h}_{b}+\tilde{S}_{b}}{\tilde{h}_{b} \tilde{S}_{b}}}}{2\left(\tilde{h}_{b}+\tilde{S}_{b}\right)^{2}} \\
+\frac{\tilde{S}_{b}\left(1-\frac{\tilde{S}_{b}}{\tilde{h}_{b}+\tilde{S}_{b}}\right)^{2} \sqrt{2 \tilde{A}_{b}} \sqrt{\frac{\tilde{h}_{b}+\tilde{S}_{b}}{\tilde{h}_{b} \tilde{S}_{b}}}}{+\frac{\tilde{A}_{b}}{\sqrt{2 \tilde{A}_{b}} \sqrt{\frac{\tilde{h}_{b}+\tilde{S}_{b}}{\tilde{h}_{b} \tilde{S}_{b}}}}}
\end{array}\right\}-
\end{aligned}
$$

Differentiating the above equation with respect to $\tilde{\delta}$, 


$$
\begin{aligned}
& \frac{\partial T \tilde{P}_{B}(\tilde{\delta})}{\partial \tilde{\delta}}=a-2 b \tilde{\delta}+b c+ \\
& \frac{b}{2 \sqrt{(a-b \tilde{\delta})}}\left\{\begin{array}{l}
\frac{\tilde{h}_{b} \tilde{S}_{b}^{2} \sqrt{2 \tilde{A}_{b}} \sqrt{\frac{\tilde{h}_{b}+\tilde{S}_{b}}{\tilde{h}_{b} \tilde{S}_{b}}}}{2\left(\tilde{h}_{b}+\tilde{S}_{b}\right)^{2}} \\
+\frac{\tilde{S}_{b}\left(1-\frac{\tilde{S}_{b}}{\tilde{h}_{b}+\tilde{S}_{b}}\right)^{2} \sqrt{2 \tilde{A}_{b}} \sqrt{\frac{\tilde{h}_{b}+\tilde{S}_{b}}{\tilde{h}_{b} \tilde{S}_{b}}}}{2}
\end{array}\right\}(6) \\
& +\frac{\tilde{A}_{b}}{\sqrt{2 \tilde{A}_{b}} \sqrt{\frac{\tilde{h}_{b}+\tilde{S}_{b}}{\tilde{h}_{b} \tilde{S}_{b}}}}
\end{aligned}
$$

Differentiating the above equation with respect to $\tilde{\delta}$,

$$
\frac{\partial^{2} T \widetilde{P}_{B}(\tilde{\delta})}{\partial \tilde{\delta}^{2}}=-2 b+
$$

$$
\frac{b^{2}}{4(a-b \tilde{\delta})^{\frac{3}{2}}}\left\{\begin{array}{l}
\frac{\tilde{h}_{b} \tilde{S}_{b}^{2} \sqrt{2 \tilde{A}_{b}} \sqrt{\frac{\tilde{h}_{b}+\tilde{S}_{b}}{\tilde{h}_{b} \tilde{S}_{b}}}}{2\left(\tilde{h}_{b}+\tilde{S}_{b}\right)^{2}\left(1-\frac{\tilde{S}_{b}}{\tilde{h}_{b}+\tilde{S}_{b}}\right)^{2} \sqrt{2 \tilde{A}_{b}} \sqrt{\frac{\tilde{h}_{b}+\tilde{S}_{b}}{\tilde{h}_{b} \tilde{S}_{b}}}} \\
+\frac{\tilde{A}_{b}}{\sqrt{2 \tilde{A}_{b}} \sqrt{\frac{\tilde{h}_{b}+\tilde{S}_{b}}{\tilde{h}_{b} \tilde{S}_{b}}}}
\end{array}\right\}
$$

Equating equation (6) equals to zero and solving the equation we will get the value of $\tilde{\delta}$, substituting this value of $\tilde{\delta}$ in equation (2) we will get the value of value of $\tilde{Q}$. Now $T \tilde{P}_{B}(\tilde{\delta})$ is concave.

\section{Vendor's Profit Policy}

When the buyer's order quantity and the selling price are adopted, the orders are received by the vendor at a known interval $\frac{\tilde{Q}}{\tilde{D}(\tilde{\delta})}$ as follows:

A vendor's average inventory can then be obtained

$$
A \tilde{I}_{v}=\frac{\tilde{Q}}{2}\left[(\tilde{n}-1)\left(1-\frac{\tilde{D}(\tilde{\delta})}{\tilde{P}}\right)+\frac{\tilde{D}(\tilde{\delta})}{\tilde{P}}\right]--
$$

$$
\begin{aligned}
& \tilde{T} P_{v}(\tilde{n})=a c-b c \tilde{\delta}-\frac{(a-b \tilde{\delta}) \tilde{A}_{v}}{\tilde{n} \tilde{Q}}- \\
& \frac{\tilde{h_{v}} \tilde{Q}}{2}\left[\tilde{n}\left(1-\frac{(a-b \tilde{\delta})}{\tilde{P}}\right)-1+\frac{2(a-b \tilde{\delta})}{\tilde{P}}\right]
\end{aligned}
$$

such that $\mathrm{n}$ is integer.

It clearly shows that $T \tilde{P}_{v}(\tilde{n})$ is concave in $\mathrm{n}$.

Optimality conditions for $n^{*}$

$$
\tilde{n}^{*}\left(\tilde{n}^{*}-1\right) \leq \frac{2(a-b \tilde{\delta}) \tilde{P} \tilde{A}_{v}}{\tilde{h}_{v} \tilde{Q}^{2}(\tilde{P}-a-b \tilde{\delta})} \leq \tilde{n}^{*}\left(\tilde{n}^{*}+1\right)--(10
$$

\section{If the buyer is free to choose his own marketing} and ordering policies $(\tilde{\delta}, \tilde{Q})$, and the vendor is free to choose its number of shipments $n$, then it is straight forward that the total system profit under individual optimization, $T \tilde{P}_{1}(\tilde{\delta}, \tilde{Q}, \tilde{n})$ is equal to the sum of buyer's and the vendor's profits.

$$
T \widetilde{P}_{1}(\tilde{\delta}, \tilde{Q}, \tilde{n})=T \widetilde{P}_{B}(\tilde{\delta})+T \tilde{P}_{v}(\tilde{n})
$$

Suppose that both parties decide to cooperate and agree to follow the jointly optimal integrated policy. The cost stemming from the purchasing price is an internal transfer of money from one supply chain member (the vendor) to another supply chain member (the buyer).

Therefore it is not a cost of the whole supply chain. The total system profit under joint optimization with shortage

$$
\begin{aligned}
& \tilde{T} P_{j}(\tilde{\delta}, \tilde{Q}, \tilde{n})=\left(a \tilde{\delta}-b \tilde{\delta}^{2}\right) \\
& -\tilde{Q}\left[\begin{array}{l}
\frac{\tilde{h}_{b} \tilde{S}_{b}^{2}}{2\left(\tilde{h}_{b}+\tilde{S}_{b}\right)^{2}} \\
+\frac{\tilde{S}_{b}\left(1-\frac{\tilde{S}_{b}}{\tilde{h}_{b}+\tilde{S}_{b}}\right)^{2}}{2} \\
+\frac{\tilde{h}_{v}}{2}\left[\begin{array}{l}
\tilde{n}\left(1-\frac{(a-b \tilde{\delta})}{\tilde{P}}\right)-1 \\
+\frac{2(a-b \tilde{\delta})}{\tilde{P}}
\end{array}\right]
\end{array}\right]
\end{aligned}
$$

$$
-\frac{1}{\tilde{Q}}\left[\tilde{A}_{b}(a-b \tilde{\delta})+\frac{\tilde{A}_{v}(a-b \tilde{\delta})}{\tilde{n}}\right]
$$

Hence the vendor's yearly profit function is , 


\section{Solution for Joint Model}

Differentiating (11) with respect to $\tilde{Q}$ we get,

$$
\frac{\partial \tilde{T} P_{j}}{\partial \tilde{Q}}=-\left[\begin{array}{l}
\frac{\tilde{h}_{b} \tilde{S}_{b}{ }^{2}}{2\left(\tilde{h}_{b}+\tilde{S}_{b}\right)^{2}} \\
+\frac{\tilde{S}_{b}\left(1-\frac{\tilde{S}_{b}}{\tilde{h}_{b}+\tilde{S}_{b}}\right)^{2}}{2} \\
+\frac{\tilde{h}_{v}}{2}\left[\tilde{n}\left(1-\frac{(a-b \tilde{\delta})}{\tilde{P}}\right)-1+\frac{2(a-b \tilde{\delta})}{\tilde{P}}\right]
\end{array}\right]
$$$$
+\frac{1}{\tilde{Q}^{2}}\left[\tilde{A}_{b}(a-b \tilde{\delta})+\frac{\tilde{A}_{v}(a-b \tilde{\delta})}{\tilde{n}}\right]--
$$

Equating (12) to zero we will get the value of $\tilde{Q}^{*}$

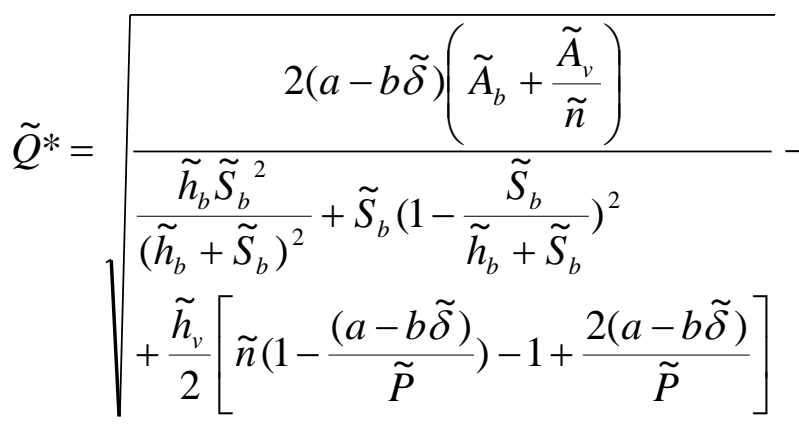

Substituting (13) in (11) we get,

$$
\tilde{T} P_{j}(\tilde{\delta}, \tilde{n})=a \tilde{\delta}-b \tilde{\delta}^{2}-
$$

$$
\sqrt{\left\{2(a-b \tilde{\delta})\left(\tilde{A}_{b}+\frac{\tilde{A}_{v}}{\tilde{n}}\right)\right\}\left\{\begin{array}{l}
\frac{\tilde{h}_{b} \tilde{S}_{b}^{2}}{\left(\tilde{h}_{b}+\tilde{S}_{b}\right)^{2}} \\
+\tilde{S}_{b}\left(1-\frac{\tilde{S}_{b}}{\left.\tilde{h}_{b}+\tilde{S}_{b}\right)^{2}}\right. \\
\tilde{h}_{v}\left[\begin{array}{c}
\tilde{n}\left(1-\frac{(a-b \tilde{\delta})}{\tilde{P}}\right)-1 \\
+\frac{2(a-b \tilde{\delta})}{\tilde{P}}
\end{array}\right]
\end{array}\right\}}
$$

$$
\left.T \tilde{P}^{1}{ }_{j}=\left\{\begin{array}{l}
2(a-b \tilde{\delta}) \\
\left(\tilde{A}_{b}+\frac{\tilde{A}_{v}}{\tilde{n}}\right)
\end{array}\right)\right\}\left\{\begin{array}{l}
\frac{\tilde{h}_{b} \tilde{S}_{b}{ }^{2}}{\left(\tilde{h}_{b}+\tilde{S}_{b}\right)^{2}} \\
+\tilde{S}_{b}\left(1-\frac{\tilde{S}_{b}}{\left.\tilde{h}_{b}+\tilde{S}_{b}\right)^{2}}\right. \\
\tilde{h}_{v}\left[\begin{array}{l}
\tilde{n}\left(1-\frac{(a-b \tilde{\delta})}{\tilde{P}}\right)-1 \\
+\frac{2(a-b \tilde{\delta})}{\tilde{P}}
\end{array}\right]
\end{array}\right\}
$$

For a given value of $\tilde{\delta}$, maximizing $T \widetilde{P}_{j}$ is equivalent to minimizing $T P^{1}{ }_{j}$

Taking the first and second partial derivatives with respect to $\tilde{n}$, and equating first order derivative to zero we obtain

$$
\tilde{n}=\sqrt{\begin{array}{l}
\tilde{A}_{v} \tilde{h}_{b} \frac{\tilde{S}_{b}{ }^{2}}{\left(\tilde{h}_{b}+\tilde{S}_{b}\right)^{2}} \\
+\tilde{A}_{v} \tilde{S}_{b}\left(1-\frac{\tilde{S}_{b}}{\tilde{h}_{b}+\tilde{S}_{b}}\right)^{2} \\
+\tilde{A}_{v} \tilde{h}_{v}\left(-1+\frac{2(a-b \tilde{\delta})}{\tilde{P}}\right) \\
\left.\frac{\tilde{A}_{b} \tilde{h}_{v}\left[1-\frac{a-b \tilde{\delta}}{\tilde{P}}\right]}{\tilde{P}}\right]
\end{array}---(16)}
$$

\section{Defuzzification of this model:}

Using Signed Distance method, we will get the crisp value of Selling price $(\delta)$, Order quantity $(Q)$, number of shipments (n) for individual and joint model, Total profit for buyer $\left(\mathrm{TP}_{\mathrm{B}}\right)$, Total profit for vendor $\left(\mathrm{TP}_{\mathrm{v}}\right)$, Total system profit under individual optimization $\left(\mathrm{TP}_{1}\right)$, the joint total profit of vendor $\left(\mathrm{TP}_{\mathrm{vj}}\right)$, the joint total profit of buyer $\left(\mathrm{TP}_{\mathrm{Bj}}\right)$, total system profit under joint optimization $\left(\mathrm{TP}_{\mathrm{j}}\right)$. For a given value of $n, \mathrm{TP}_{\mathrm{j}}$ can be written as

$$
T P_{j}(D)=m_{1} D+m_{2} D^{2}-\sqrt{m_{3} D+m_{4} D^{2}}
$$




$$
\begin{aligned}
& m_{1}=\frac{a}{b} \\
& m_{2}=\frac{-1}{b}
\end{aligned}
$$$$
m_{3}=2\left(K_{b}+\frac{K_{v}}{n}\right)\left\{\begin{array}{l}
h_{b}\left(\frac{S_{b}}{\left(h_{b}+S_{b}\right)}\right)^{2} \\
+\frac{h_{v}}{n}\left(\frac{S_{v}}{S_{v}+h_{v}}\right)^{2} \\
+S_{b}\left(1-\frac{S_{b}}{h_{b}+S_{b}}\right)^{2}-S_{v}\left(1-\frac{S_{v}}{h_{v}+S_{v}}\right)^{2}(n-1)
\end{array}\right\}
$$$$
m_{4}=\frac{2}{P}\left(K_{b}+\frac{K_{v}}{n}\right) S_{v}\left(1-\frac{S_{v}}{S_{v}+h_{v}}\right)^{2}(2-n)
$$

$$
\text { and } \mathrm{D}(\delta)=\mathrm{a}-\mathrm{b} \delta
$$

There is a one to one relationship between price and demand. Therefore, we base our analysis on the identification of the optimal value of demand, rather than the optimal value of price. The first and second partial derivative of $\mathrm{TP}_{\mathrm{j}}(\mathrm{D})$, with respect to $\mathrm{D}$ are as follows.

$$
\frac{\partial T P_{j}(D)}{\partial D}=m_{1}+2 m_{2} D-\frac{m_{3}+2 m_{4} D}{2 \sqrt{m_{3} D+m_{4} D^{2}}}
$$

\section{Numerical example:}

We consider an example with the following data:

$$
\begin{aligned}
& \tilde{P}=(3100,3200,3300) / \text { year } \\
& \tilde{K}_{b}=(R s .20, R s .25, R s .30) / \text { order } \\
& \tilde{h}_{b}=(R s .4, R s .5, R s .6) / \text { unit / year } \\
& \tilde{S}_{b}=(R s .8, R s .9, \text { Rs.10 }) / \text { unit }
\end{aligned}
$$

$$
\frac{\partial^{2} T P_{j}(D)}{\partial D^{2}}=2 m_{2}+\frac{m^{2}{ }_{3}}{4}\left(m_{3} D+m_{4} D^{2}\right)^{\frac{-3}{2}}
$$

\section{Case 1: $\mathrm{n}=1$}

Hence $\mathrm{m}_{4}>0$, therefore there are two saddle points, $\mathrm{SP}_{1}$ and $\mathrm{SP}_{2}$. The total profit function is convex when $\mathrm{SP}_{1}<\mathrm{D}<\mathrm{SP}_{2}$, and is concave when $\mathrm{D} \leq \mathrm{SP}_{1}$ or $\mathrm{D} \geq \mathrm{SP}_{2}$. The optimal value of the demand is then

$\mathrm{D}^{*}=\mathrm{LO}_{1}$ if $\mathrm{LO}_{1}<\mathrm{a}$, and it is $\mathrm{D}^{*}=\mathrm{a}$ if $\mathrm{LO}_{1} \geq \mathrm{a}$.

Case 2: $\mathbf{n = 2}$

Hence $\mathrm{m}_{4}=0$, and therefore there is a saddle point, $S P=\frac{b^{\frac{2}{3}} m^{\frac{1}{3}} 3}{4}$ the total profit function is convex when D $\mathrm{SP}$, and is concave when $\mathrm{D} \geq \mathrm{SP}$. Because the total profit function is zero at $\mathrm{D}=0$, there is no more than one local optimal amount for the demand. The optimal value of the demand is then

$\mathrm{D}^{*}=\mathrm{LO}_{2}$ if $\mathrm{LO}_{2}<\mathrm{a}$, and it is $\mathrm{D}^{*}=\mathrm{a}$ if $\mathrm{LO}_{2} \geq \mathrm{a}$.

\section{Case 3: $n \geq 3$}

Hence, $\mathrm{m}_{4}<0$, and therefore there are two saddle points, $\mathrm{SP}_{1}$ and $\mathrm{SP}_{2}$. The total profit function is concave when $\mathrm{SP}_{2}<\mathrm{D}<\mathrm{SP}_{1}$, and it is convex when $\mathrm{D} \leq \mathrm{SP}_{2}$ or $\mathrm{D} \geq$ $\mathrm{SP}_{1}$. Moreover, $\mathrm{m}_{3}, \mathrm{t}>0$ and thus $\mathrm{SP}_{1}>0$ and $\mathrm{SP}_{2}>0$ The optimal value of the demand is then $\mathrm{D}^{*}=\mathrm{LO}_{3}$ if $\mathrm{LO}_{3}<\mathrm{a}$, and it is $\mathrm{D}^{*}=\mathrm{a}$ if $\mathrm{LO}_{3} \geq \mathrm{a}$.

As no closed form solution exists for the local optimal values of the demand, we use numerical method to find $\mathrm{LO}_{\mathrm{i}}, \mathrm{i}=1,2$, 3, .

$$
\begin{aligned}
& \tilde{K}_{v}=(R s .300, R s .400, R s .500) / \text { setup } \\
& \tilde{h}_{v}=(R s .3, R s .4, R s .5) / \text { unit } / \text { setup } \\
& \text { a=1500, b= 10, c= Rs.5/unit } \\
& \tilde{S}_{v}=(R s .80, R s .85, R s .90) / \text { unit }
\end{aligned}
$$

We analyze the effect demand's price sensitivity. The effect is evaluated by the impact on the benefits of vendor-buyer coordination as well as impact on the decision variables.

$\mathrm{TP}_{\mathrm{j}}$ and $\mathrm{TP}_{1}$ represent the total system profit under joint and individual optimization.

Joint total profit allocated to the buyer and the vendor as follows (see Ouyang et. al., [9], Wu and Ouyang [10],

$$
T P_{v j}=\frac{T P_{v}(n)}{T P_{1}} T P_{j} a n d T P_{B j}=\frac{T P_{B}(n)}{T P_{1}} T P_{j}
$$




\begin{tabular}{|c|c|c|c|c|c|c|}
\hline \multicolumn{7}{|c|}{ Decision Variables under individual optimization (fuzzy environment) } \\
\hline $\mathrm{b}$ & $\delta$ & Q & $\mathrm{n}$ & $\mathrm{TPv}$ & TPB & TP1 \\
\hline 10 & $\begin{array}{l}(77.3647, \\
77.5463, \\
77.7284)\end{array}$ & $\begin{array}{c}(69.397, \\
85.088, \\
104.102)\end{array}$ & $(5,5,5)$ & $\begin{array}{l}(1708.596, \\
2381.424, \\
2867.229)\end{array}$ & $\begin{array}{l}(51507.994, \\
52162.444, \\
52636.900)\end{array}$ & $\begin{array}{l}(53216.59, \\
54543.868, \\
55504.129)\end{array}$ \\
\hline 20 & $\begin{array}{l}(39.9611, \\
39.9519, \\
39.9807)\end{array}$ & $\begin{array}{l}(74.854, \\
104.235, \\
144.994)\end{array}$ & $(5,5,5)$ & $\begin{array}{l}(1332.563, \\
2269.909, \\
2844.097)\end{array}$ & $\begin{array}{l}(23722.093, \\
24164.336, \\
24371.016)\end{array}$ & $\begin{array}{l}(25054.656, \\
26434.245, \\
27215.113)\end{array}$ \\
\hline 30 & $\begin{array}{l}(19.2655, \\
19.2656, \\
19.2656)\end{array}$ & $\begin{array}{l}(85.885, \\
119.547, \\
166.316)\end{array}$ & $(5,5,5)$ & $\begin{array}{l}(2206.478, \\
3243.344, \\
3881.656)\end{array}$ & $\begin{array}{c}(12276.7895 \\
12768.419, \\
12989.613)\end{array}$ & $\begin{array}{l}(14483.267, \\
16011.763, \\
16871.269)\end{array}$ \\
\hline 40 & $\begin{array}{l}\text { (12.8106, } \\
12.8118, \\
12.8118)\end{array}$ & $\begin{array}{l}\text { (88.883, } \\
\text { 123.720, } \\
172.126)\end{array}$ & $(5,5,5)$ & $\begin{array}{c}(2475.053, \\
3538.403, \\
4192.93)\end{array}$ & $\begin{array}{c}(6806.1032, \\
7316.015, \\
7545.2845)\end{array}$ & $\begin{array}{l}\text { (9281.156, } \\
10854.418, \\
11738.215)\end{array}$ \\
\hline
\end{tabular}

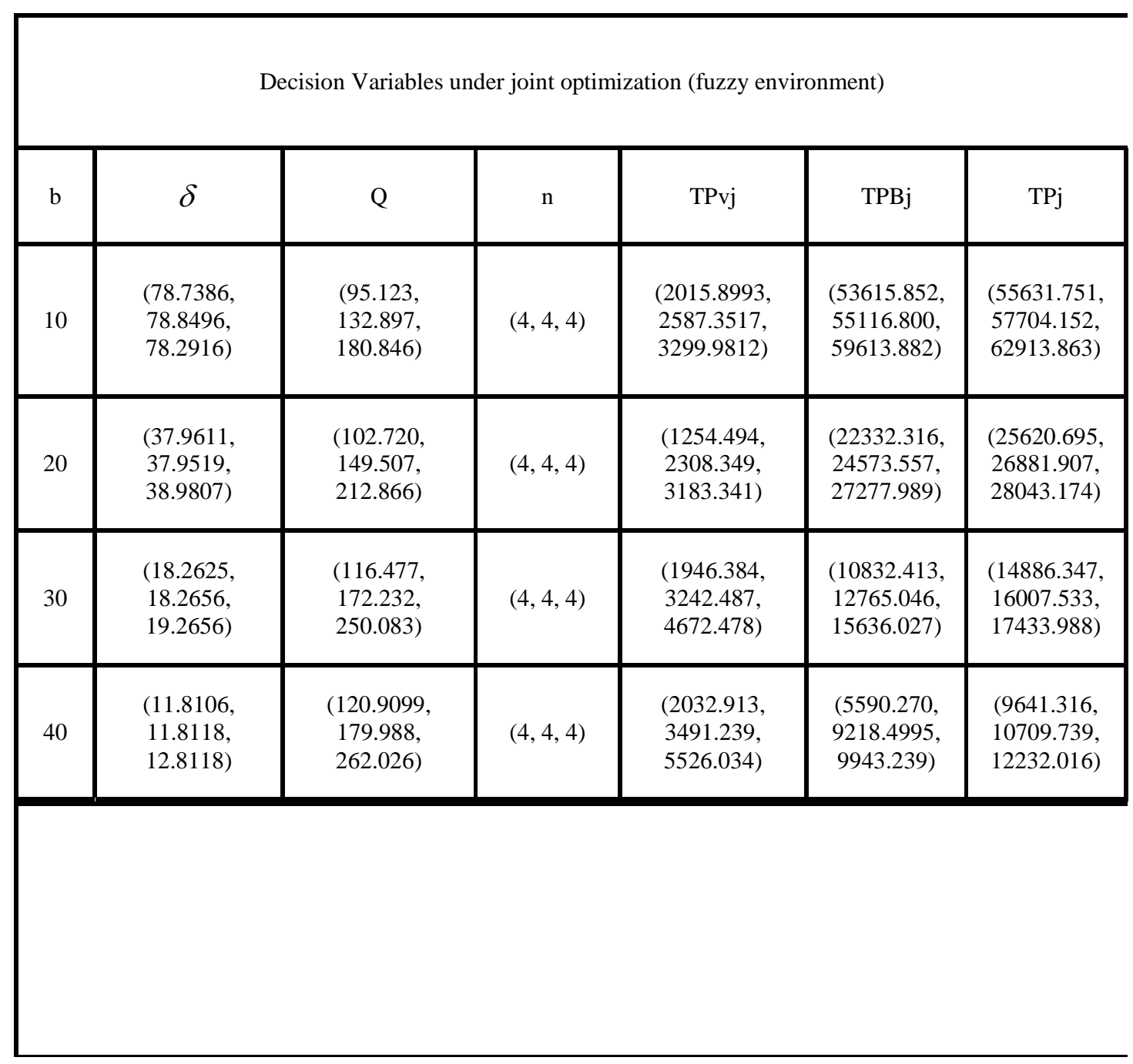




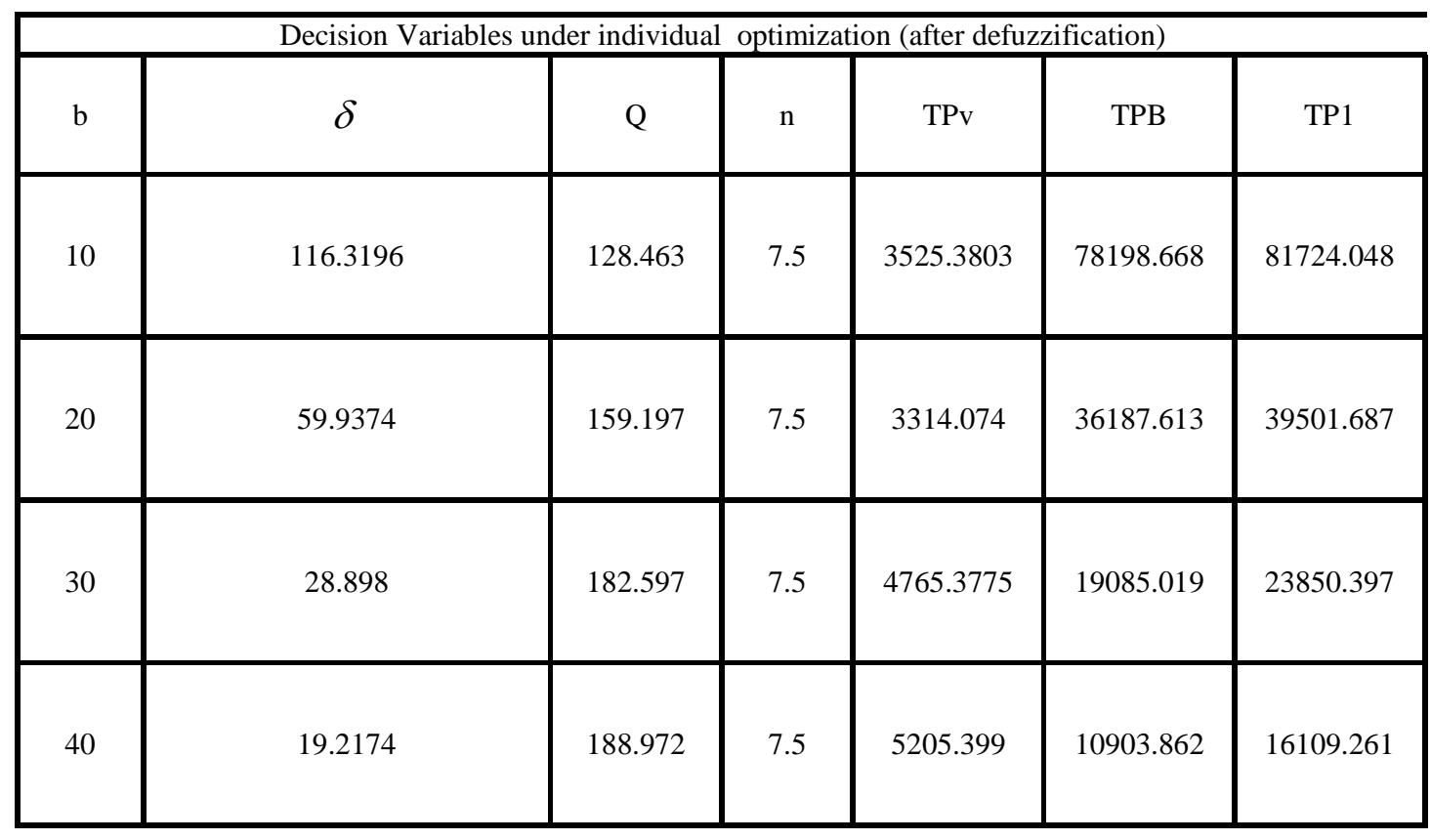

\begin{tabular}{|c|c|c|c|c|c|c|}
\hline \multicolumn{7}{|c|}{ Decision Variables under joint optimization (after defuzzification) } \\
\hline $\mathrm{b}$ & $\delta$ & $\mathrm{Q}$ & $\mathrm{n}$ & $\mathrm{TPvj}$ & $\mathrm{TPBj}$ & $\mathrm{TP} 1 \mathrm{j}$ \\
\hline 10 & 118.2647 & 201.8913 & 6 & 3916.3218 & 83424.2335 & 87340.555 \\
\hline 20 & 57.1874 & 228.4035 & 6 & 3417.808 & 36976.133 & 40297.874 \\
\hline 30 & 27.648 & 263.872 & 6 & 4897.203 & 19382.156 & 24087.617 \\
\hline 40 & 17.9674 & 275.722 & 6 & 5380.976 & 13101.897 & 16178.072 \\
\hline
\end{tabular}

\section{CONCLUSION}

This paper addresses an inventory problem under fuzzy cost with allowable shortage for buyer. In this paper joint total profit of both buyer and vendor are calculated. Our venture of this paper is to get more profit for buyer and vendor in co ordination comparatively with their individual profits without co - ordination. We have developed the model which is more suitable for real life situations. We have achieved in this model by obtaining more profit for the required model consequently our optimum profit for the joint model is more than the profit of the model proposed by [8].

\section{REFERENCES}

[1]. Inventory systems by Eliezer Naddor

[2] Fuzzy sets and logics by Zadeh
[3] Goyal, S.K., 1976. An integrated inventory model for a single supplier-single customer problem. International journal of Production Research 15(1), 107-111.

[4] Goyal, S.K., 1988. A joint economic-lotsize model for purchaser and vendor; a comment Decision science 19, 236-241.

[5] Lau, A.H.L., Lau, H.S., 2003. Effects of a demandcurve's shape on the optimal solutions of a multiechelon inventory/pricing model. European journal of Operational Research 147, 530-548.

[6] Ray. S. Gerchek. Y., Jewkers. E. M., 2005. Join pricing and inventory policies for make - to - stock products with deterministic price sensitive demand. International Journal of Production Economics 97, 143-158. 
[7] Mohsen S. Sajadieh., Mohammad R. Akbari Jokar., 2009. Optimizing shipment, ordering and pricing policies in a two-stage supply chain with price-sensitive demand. Transportation Research Part E 45, 564-571.

[8] A. Nagoor Gani, G. Sabarinathan., 2012. A new method for solving two stage supply chain fuzzy inventory problem., applied mathematical sciences,60, 2963-2978
[9] Ouyang, L., Wu., K., Ho, C., 2004. Integrated vendor buyer co operating models with stochastic demand in controllable lead time International Journal of Production Economics 92, 255-256.

[10] Wu. k. Ouyang, L., 2003 An integrated single vendor single buyer inventory system with shortage derived algebraically, Production Planning and Control 14(6), $555-561$ 